\title{
DCF LEARN AND PERformanCE ANALYSis OF 802.11B WIRELESS NETWORK
}

\author{
Mingming $\mathrm{Li}^{1}$, Biao Huang ${ }^{2}$, Haiyang Liu ${ }^{1}$ and Miao Yang ${ }^{1}$ \\ ${ }^{1}$ Spectrum Management Research Division, SRRC, Beijing, China \\ ${ }^{2}$ Spectrum Engineering Department, SRRC, Beijing, China
}

\begin{abstract}
Though WLAN wireless network has been widely deployed as the main split-flow deployment of the communication network, little study emphasizes its performance as WLAN protocols were only designed for the public communicating conveniently with each other. Actually that too much wireless access points assembling together will cause self-interference to the whole WLAN network. This paper investigates the distributed coordination function (DCF) learn and the performance study of $802.11 \mathrm{~b}$ networks. Firstly, our study illustrates the performance of its MAC layer and its fairness issues related to DCF. Next we propose the details which should be paid attention to in deploying network services. Then, performance analyses are evaluated by simulation and real test for a dense wireless network. Our main goal is to give proposals to network operators how to design a WLAN network more standardized and orderly.
\end{abstract}

\section{KEYWORDS}

WLAN network, 802.11b, Performance Analysis, Distributed Coordination Function, Fairness

\section{INTRODUCTION}

Although IEEE 802.11 standard series include802.11, 802.11b, 802.11a, 802.11g, 802.11n, $802.11 \mathrm{AC}$, etc., $802.11 \mathrm{~b}$ is one most-widely deployed version as it originally appeared. This technology operates in the $2.4 \mathrm{GHz}$ ISM (Industrial, Scientific, and Medical) radio spectrum with signal bandwidth $20 \mathrm{MHz}$. Complementary Code Keying(CCK) or Direct sequence spread spectrum (DSSS) and carrier sense multiple access with collision avoidance (CSMA/CA) are respectively used as key techniques both in physical layer and MAC layer, supporting variable data rates $1,2,5.5$ and $11 \mathrm{Mbps}$.

In IEEE Std. 802.11b-1999[1], the CCK modulation used by $802.11 \mathrm{~b}$ transmits data in symbols of eight chips, where each chip is a complex QPSK bit-pair at a chip rate of 11Mchip/s. In 5.5 $\mathrm{Mbit} / \mathrm{s}$ and $11 \mathrm{Mbit} / \mathrm{s}$ modes respectively 4 and 8 bits are modulated onto the eight chips of the symbols $c_{0}, \ldots, c_{7}$, where $c=c_{0}, \ldots, c_{7}=\left(e^{j\left(\phi_{1}+\phi_{2}+\phi_{3}+\phi_{4}\right)}, e^{j\left(\phi_{1}+\phi_{2}+\phi_{4}\right)},-e^{j\left(\phi_{1}+\phi_{4}\right)}, e^{j\left(\phi_{1}+\phi_{2}+\phi_{3}\right)}\right.$, $\left., e^{j\left(\phi_{1}+\phi_{3}\right)},-e^{j\left(\phi_{1}+\phi_{2}\right)}, e^{j \phi_{1}}\right)$ and $\phi_{1}, \phi_{2}, \phi_{3}, \phi_{4}$ are determined by the bits being modulated. Then $802.11 \mathrm{~b}$ has a maximum raw data rate of $11 \mathrm{Mbit} / \mathrm{s}$. As we know, $802.11 \mathrm{~b}$ uses the same CSMA/CA media access method in its MAC layer and DCF is one coordination function for its channel access. Within DCF, there are two ways to access the MAC layer. One way is that each successful transmission follows the so-called 4-way handshake protocol of RTS_CTS_DATA_ACK. Once hearing RTS, the neighbour nodes in set their NAVs to the duration mentioned in RTS. After hearing CTS, the nodes in the vicinity of receiver set their NAVs to the duration mentioned in CTS. Then each DATA packet is preceded. This causes in 
establishment of channel reservation till the time the ACK is sent back to the sender. The timeline for DCF message exchanges is shown in Figure 1[1]. The other way is so-called CTS_self of DATA_ACK, which is quite simple than 4-way handshake protocol. Due to CSMA/CA protocol overhead, in practice the maximum $802.11 \mathrm{~b}$ throughput that an application can achieve is about 5.9 Mbit/s using TCP and 7.1 Mbit/s using UDP. Many simulation results and test results will survey this statement.

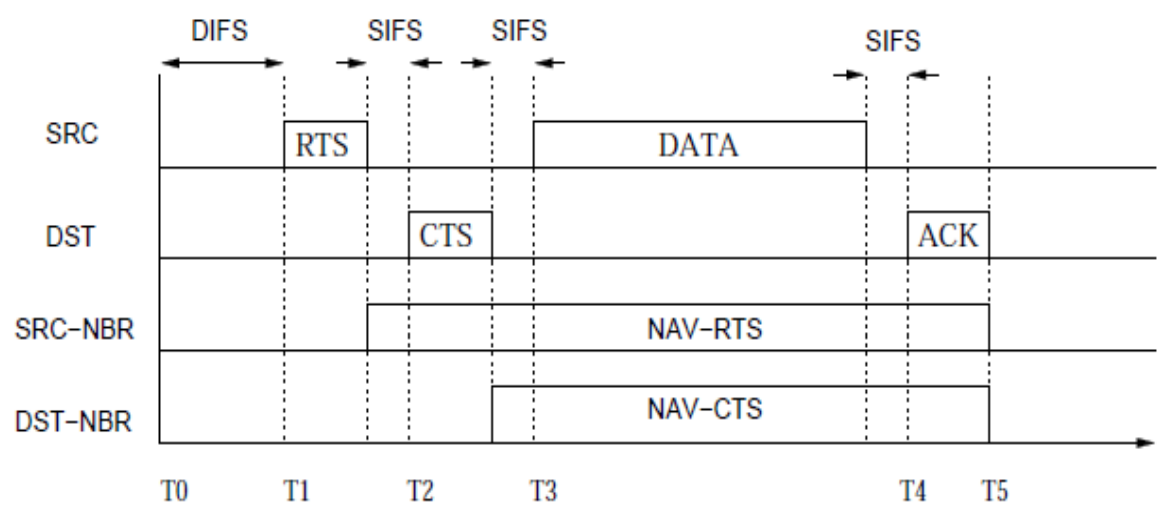

Figure 1. Message exchanging in Distributed Coordination Function

This paper mainly gives study illustrating the fairness issues of DCF and the performance of $802.11 \mathrm{bs}$ MAC layer. Then some details are referred to give proposals to network operators how to design a WLAN network more standardized and orderly. In Section 2, we give a mathematical definition and a comparison of the fairness issues related to 802.11b MAC layer. Section 3 gives a particular introduction to $802.11 \mathrm{~b}$ performance analysis through simulation and test methods. At the end, some proposals are mentioned to network operators and conclude our study.

\section{Performance and Fairness of 802.11b's MAC layer}

\subsection{Scenario 1: Only One UE in a BSS}

A BSS (Basic Service Set) composed one AP is the basic structural unit of WLAN network. We presume a simple WLAN network including only one UE in a BSS. When the user accesses the network, none will race to control the channel with it. The throughput can be modelled as:

$$
R_{1}=\frac{\text { DATA length }}{\text { DATA Delay }}
$$

One ordinary frame with the length 2346 bytes in MAC layer looks like the follow. Done like in IEEE Std. 802.11b [1], in a simple WLAN network, the DATA can be chosen as the MSDU (MAC layer service data unit). Correspondingly, the DATA delay is a time slot. As referred in the part one, in DCF, each successful transmission follows the 4-way handshake protocol or CTS_self. Then we can get:

$$
\begin{gathered}
\text { Delayer } M S D U_{\text {Handshake_4 }_{4}}=\left(T_{D I F S}+T_{\text {SIFS }}+T_{B O}+T_{R T S}+T_{C T S}+T_{A C K}+T_{D A T A}\right) \\
\text { Delayer } M S D U_{C T S_{-} \text {self }}=\left(T_{D I F S}+T_{S I F S}+T_{B O}+T_{A C K}+T_{D A T A}\right)
\end{gathered}
$$


International Journal of Computer Networks \& Communications (IJCNC) Vol.5, No.6, November 2013

As described in [2], the upper delay parameters are constant presented in table 1. With formula 1, 2, 3 and table I, the R1 can be plotted as figure 2, which shows the maximum throughput of scenario 1 is about 7.1 Mbps for IEEE Std. $802.11 \mathrm{~b}$. The result is the maximum $802.11 \mathrm{~b}$ throughput using UDP retrospect in Part 1. Even this conclusion will be confirmed by simulation and test results again Part 3.

Table 1. Time of various frames in IEEE STD 802.11

\begin{tabular}{|c|c|c|c|c|c|c|c|}
\hline \multirow{2}{*}{ Scheme } & \multicolumn{7}{|c|}{ Time of delay $(m s)$} \\
\hline & DIFS & SIFS & $\mathrm{BO}$ & RTS & CTS & $\mathrm{ACK}$ & DATA \\
\hline \multicolumn{8}{|c|}{ CTS_self } \\
\hline HR-5.5 & 50 & 10 & 310 & N/A & N/A & 304 & $192+8 *(34+\mathrm{MSDU}) / 5.5$ \\
\hline HR-11 & 50 & 10 & 310 & N/A & N/A & 304 & $192+8 *(34+\mathrm{MSDU}) / 11$ \\
\hline \multicolumn{8}{|c|}{ RTS/CTS } \\
\hline HR-5.5 & 50 & $10 * 3$ & 310 & 352 & 304 & 304 & $192+8 *(34+\mathrm{MSDU}) / 5.5$ \\
\hline HR-11 & 50 & $10 * 3$ & 310 & 352 & 304 & 304 & $192+8 *(34+\mathrm{MSDU}) / 11$ \\
\hline
\end{tabular}

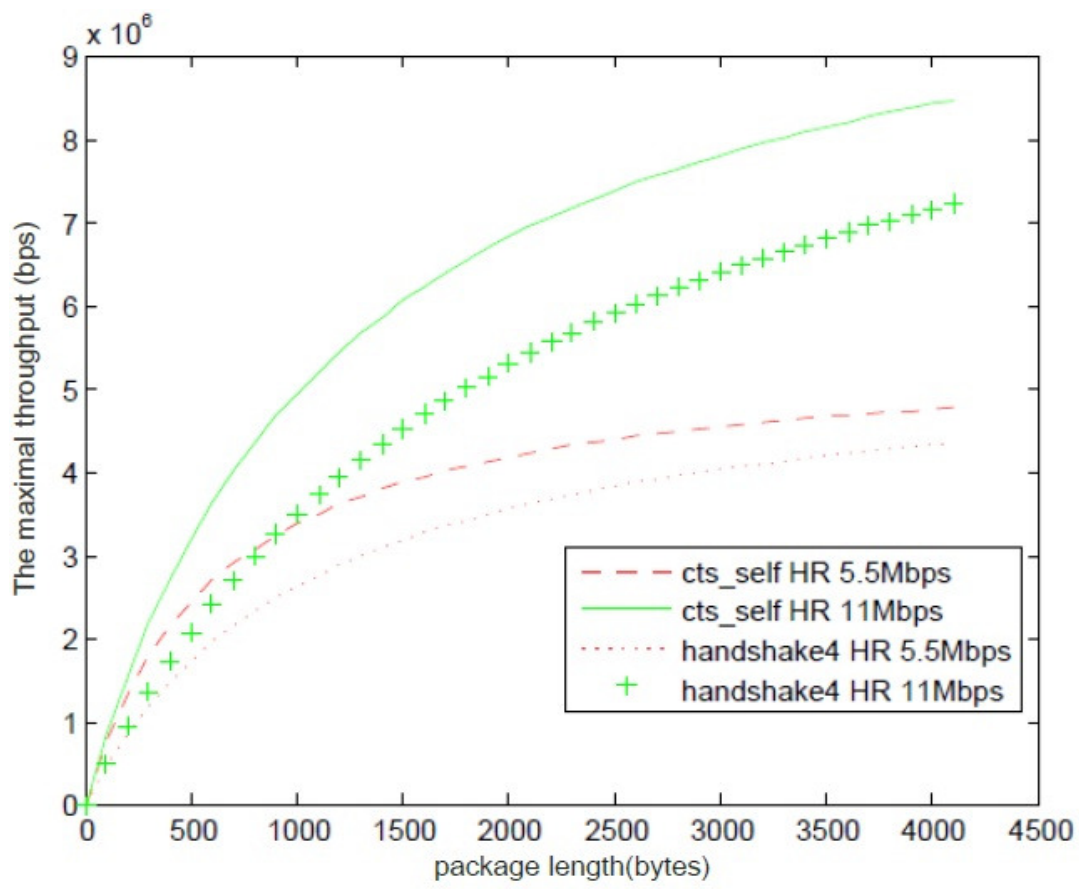

Figure 2. The compare of CTS_self's maximal throughput vs Handshake_4's

\subsection{Scenario 2: $N$ UEs in a BSS}

Also, presumption is made as $N$ UEs $(N>1)$ competing the same channel in a BSS and assuming $N$ UEs randomly located in a circle with AP as the centre. With DCF, UEs in this scene will execute back-off mechanism [1]. G. Bianchi, in whose article [3], modelled this competing process as two-dimensional discrete time Markov chain. Making use of this thesis, the authors intend to prove the fairness of DCF at first. Given q is the probability that one UE will transmit a package and $P_{t}$ is the probability that at least one UE transmits the packet in the 
considered slot time; we can write as $P_{t}=1-(1-q)^{N}$ Assuming that any $i$ th UE and the $j$ th UE have the similar wireless condition to access AP, AP will receive the same power both from any UE. That means to any UE in the network, its transmitting probability is $P_{t} / N$. In this situation, DCF proves its fairness to any UE [3], [4], [5], [6]. G. Bianchi in [3] gave out the throughput analysis by equation 13. Xiang Ling and Kwan Lawrence Yeung calculated the maximum throughput of the network with N APs with this method in [4].

Table 2. Various parameters of IEEE STD 802.11HR/DSSS technology

\begin{tabular}{|l|l|}
\hline a slot time & $20 \mu \mathrm{s}$ \\
\hline a Air Propagation Delay & $1 \mu \mathrm{s}$ \\
\hline a MAC Processing Delay & $\leq 2 \mu \mathrm{s}$ \\
\hline a MPDU length & $14 \leq x \leq\left(2^{12}-1\right)$ \\
\hline$w_{\min }$ & 31 \\
\hline$w_{\max }$ & 1023 \\
\hline
\end{tabular}

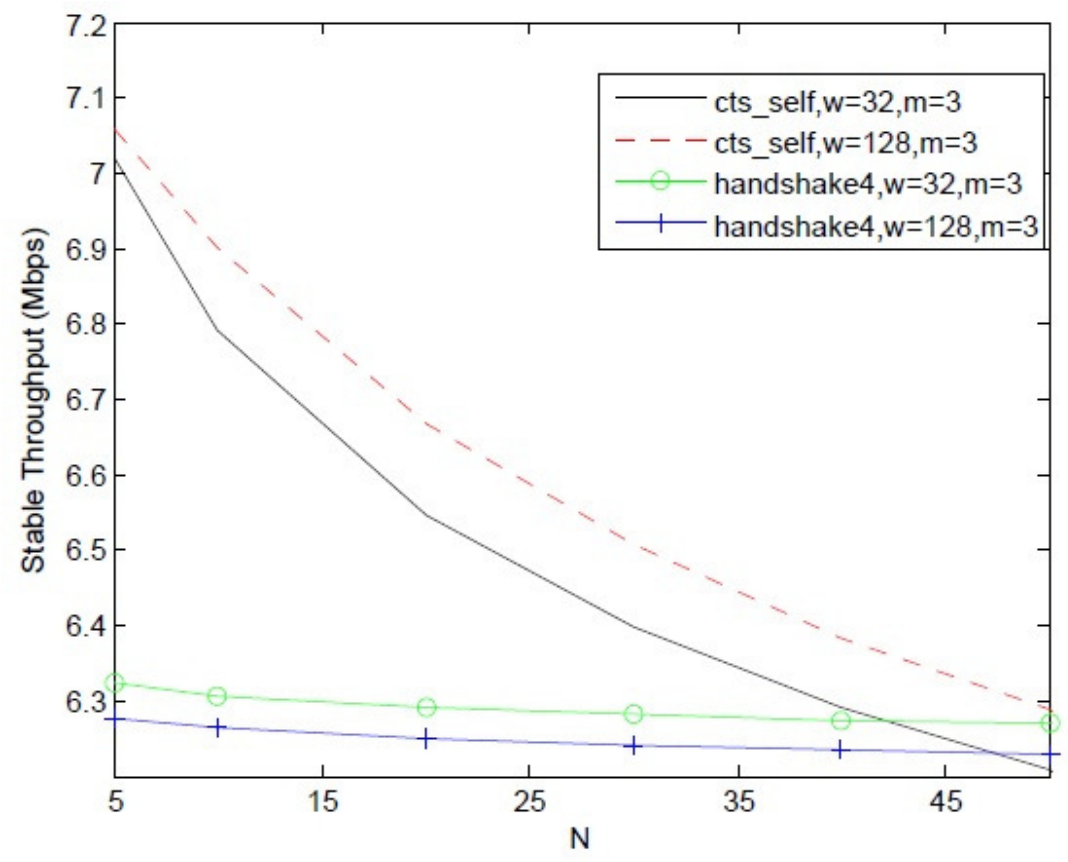

Figure 3. The contrast of CTS_self 's stable throughput and Handshake_4's

Using the parameters described in table 2, we verify G. Bianchi's results with $C W_{-}$min $=32$ or 128 and find that the WLAN networks maximal stable throughput is near $7.05 \mathrm{Mbps}$, but the maximal stable CTS_throughput is less than ACK_DATA_throughput when $N<40$ in figure 3. Under the scenario of WLAN network with multiple users using the same channel, we suggest network operators enable the CTS/RTS component on all APs when $N>40$. 


\section{Performance Analysis of 802.11b Wireless Network With SimUlation AND TEST}

\subsection{SIMULATION A: A BSS's Throughput of MAC layer with One User}

As mentioned in scenario 1, the length of the biggest data frame in MAC layer is 2304 bytes while no fragmentation configuration on the network nodes. Meanwhile setting the transmitting package length in MAC layer being equal to the length of the biggest data frame, we can plot the maximal throughput and the time delay of 802.11b MAC layer with the simulation seeds 256 and time length 3 minutes in figure 4.The 4-way handshake protocol or CTS_self protocol, its stable throughput by simulation is close to the theoretical value in figure 2 or $3,7.1 \mathrm{Mbps}$ or $5.5 \mathrm{Mbps}$ respectively. As obviously shown that more packages are transmitted in the 4-way handshake protocol in figure 1, the time delay of the 4-way handshake protocol is much longer than CTS_self protocol. This may help in claiming that there is no advantage to improve system performance by using the 4-way handshake protocol when a wireless network is lack of users in the same channel.

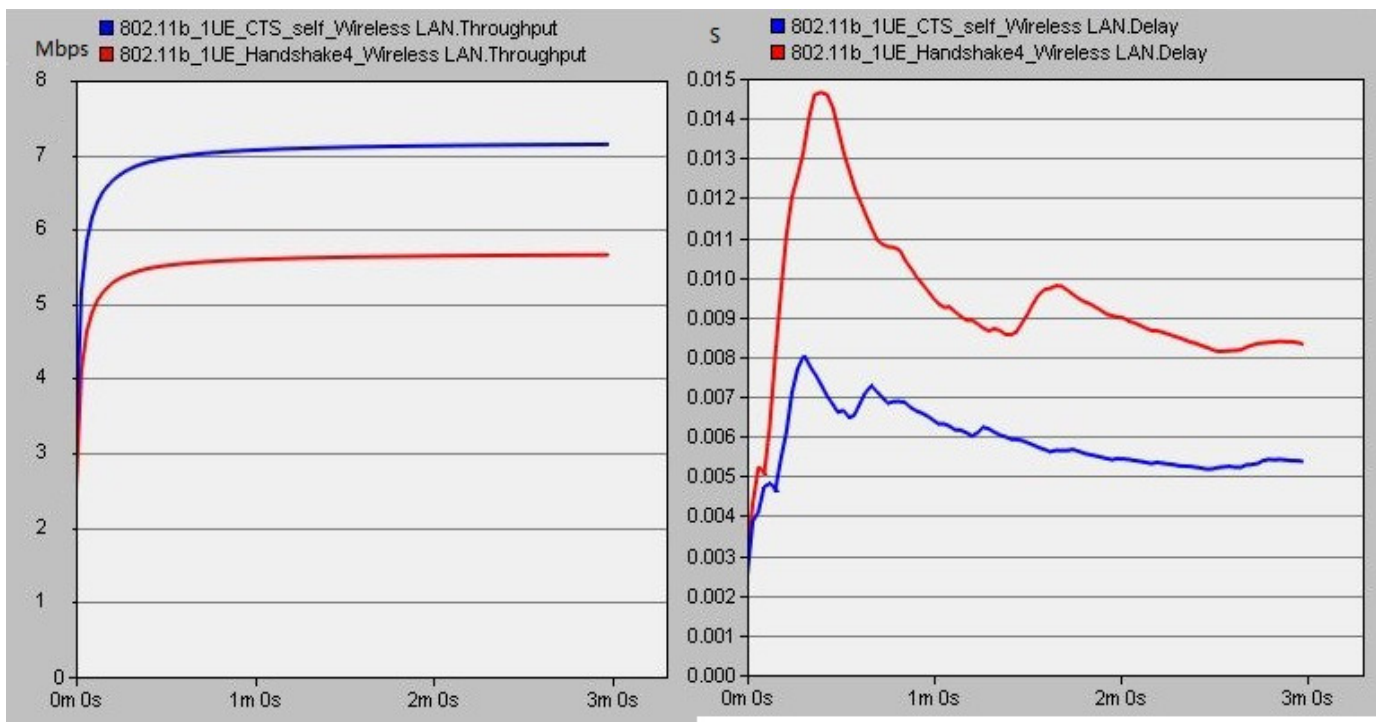

Figure 4. A BSS's throughput and delay of MAC layer with $1 \mathrm{UE}$

\subsection{SIMULATION B: DCF Fairness and BSS's Throughput of MAC layer with N Users}

Now, it is turn to prove fairness of DCF by simulation mentioned in scenario 2. Announcing a wireless network like the figure 5, ten users are located the same distance far away the centre AP downloading package of $100 \mathrm{~K}$ bytes in every $0.169 \mathrm{~s}$. The users transmitting speed is $11 \mathrm{Mbps}$ and CTS_self protocol are used in MAC layer.

Table 3. Parameters setup in simulation B

\begin{tabular}{|l|l|l|l|l|}
\hline Parameters & $\begin{array}{l}\text { BSS } \\
\text { Number }\end{array}$ & $\begin{array}{l}\text { AP } \\
\text { Number }\end{array}$ & $\begin{array}{l}\text { UE } \\
\text { Number }\end{array}$ & service \\
\hline Values & 1 & 1 & 10 & FTP \\
\hline Parameters & DATA & Time & Power & Access \\
\hline
\end{tabular}




\begin{tabular}{|l|l|l|l|l|}
\hline & Length & & & Mechanism \\
\hline Values & $100 \mathrm{~KB}$ & $0.169 \mathrm{~s}$ & $100 \mathrm{~mW}$ & CTS_self \\
\hline
\end{tabular}



Figure 5. The scenario of a BSS with N UE

In the upper case, it is to say that any UE can access AP with the similar wireless condition shown in the table 3; AP will receive the same power both from any UE. Without other limitation, we can get ten users total throughput is about $4 \mathrm{Mbps}$ and every user is about $0.4 \mathrm{Mbps}$ from figure 6 . And also, the time delay of every user confirms that every user nearly has the same chance to access the same channel. In retrospection BSS's throughput of MAC layer with N users, we have verified that the 4-way handshake protocol is not fit for less than 40 users within a wireless network. With this reason, we plot the maximal stable throughput only for CTS_self protocol with the length of the biggest data frame 2304 bytes in MAC layer on every nodes and $C W_{-} \min =32$.

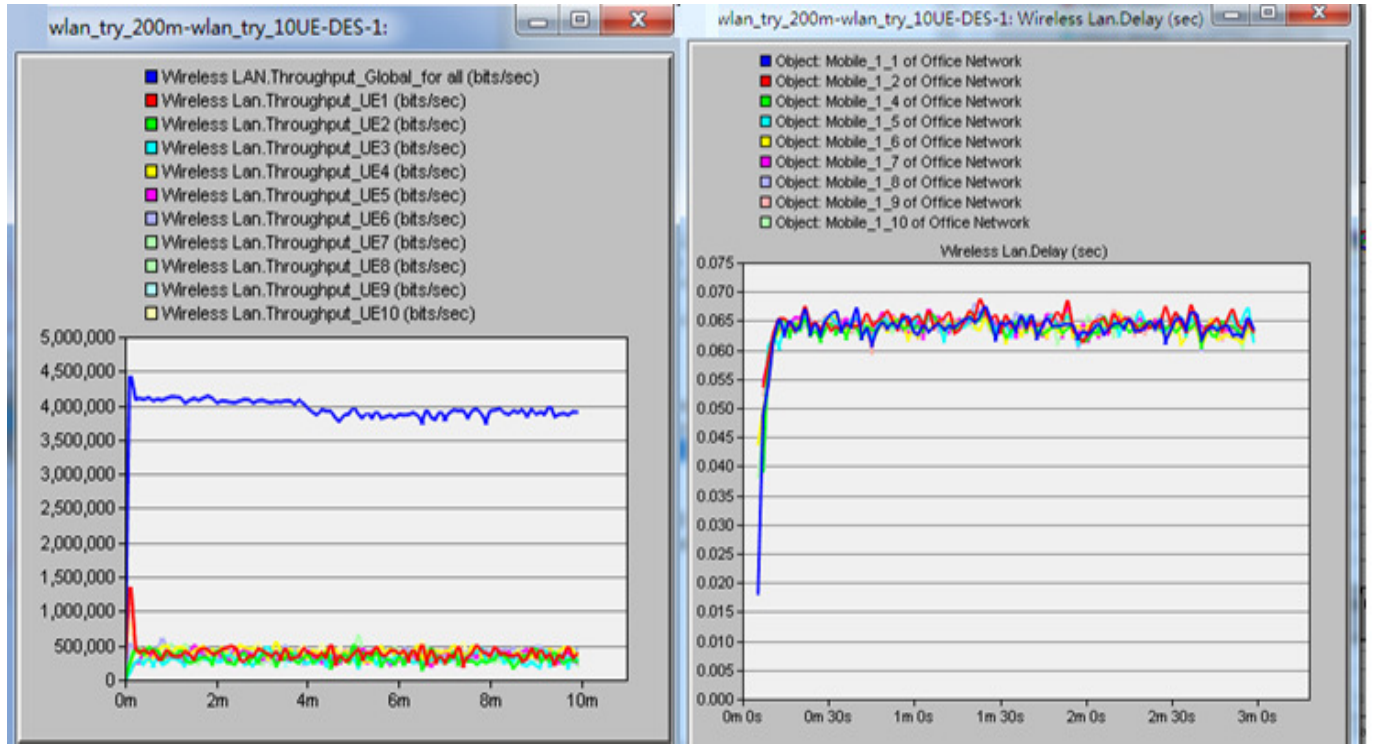

Figure 6. The throughput of a BSS with $10 \mathrm{UE}$

Figure 7 shows the stable throughput by simulation is close to the theoretical value in figure 3 when the user number is $N=5,10,20,30,40,50$. However, the stable throughput by simulation 
is less than its theoretical when $N=30$. As the user number increasing, the occurrence possibility in an interval of time of more users increasing, collision between different users burst the same time. Until $N=40$, the maximal stable throughput of MAC layer is also bigger than $5.5 \mathrm{Mbps}$ associated to multiuser diversity gain. Compared the throughput line of $N=50$ with others, we affirm the constraint of DCF that the user number $N$ is better no more than 40 within a BSS.

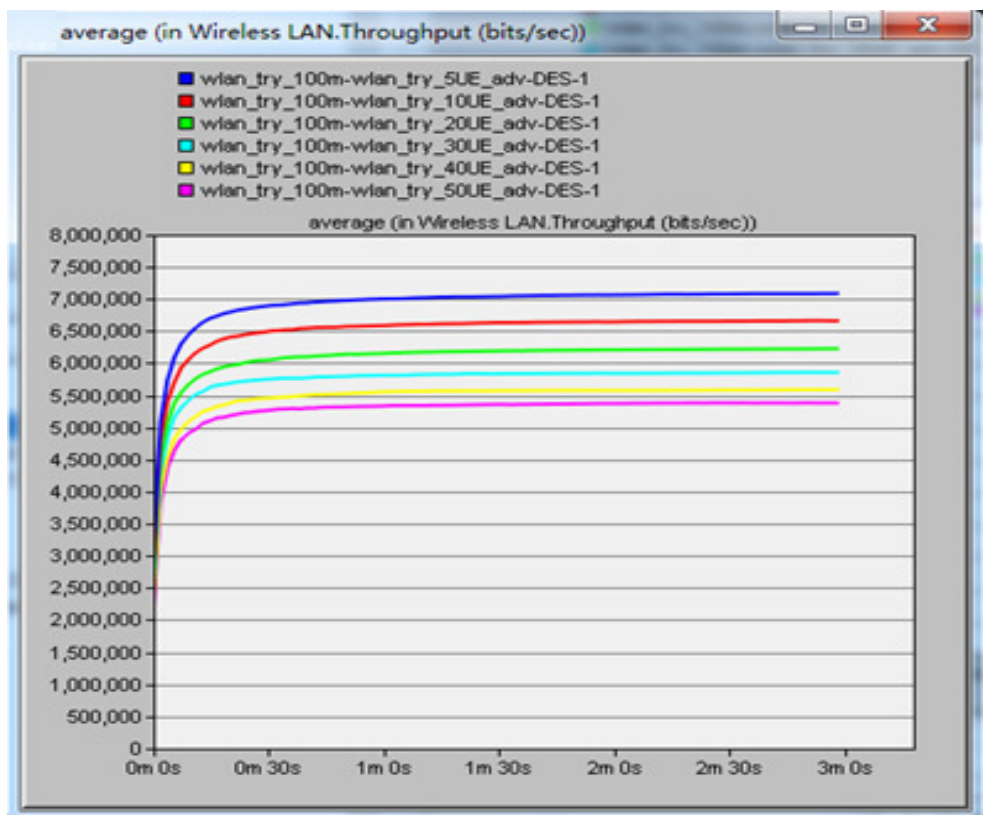

Figure 7. The throughput contrast of a BSS with different Users

\subsection{SIMULATION C: A BSS's Throughput with TCP Protocol}

Reference [7] had learned the method to improve the network's throughput with TCP protocol; the following simulation is given out to show the wireless networks performance of application layer in fact. Supposing that a BSS contains 10, 20, 30, 40, 50 users respectively and all users are downloading package of $1 \mathrm{MB}$ with the transmitting speed $11 \mathrm{Mbps}$, we optimally configure the network parameters and use CTS_self protocol by the MAC layer accessing, and other parameters are the same as values shown in table 3 . 
International Journal of Computer Networks \& Communications (IJCNC) Vol.5, No.6, November 2013

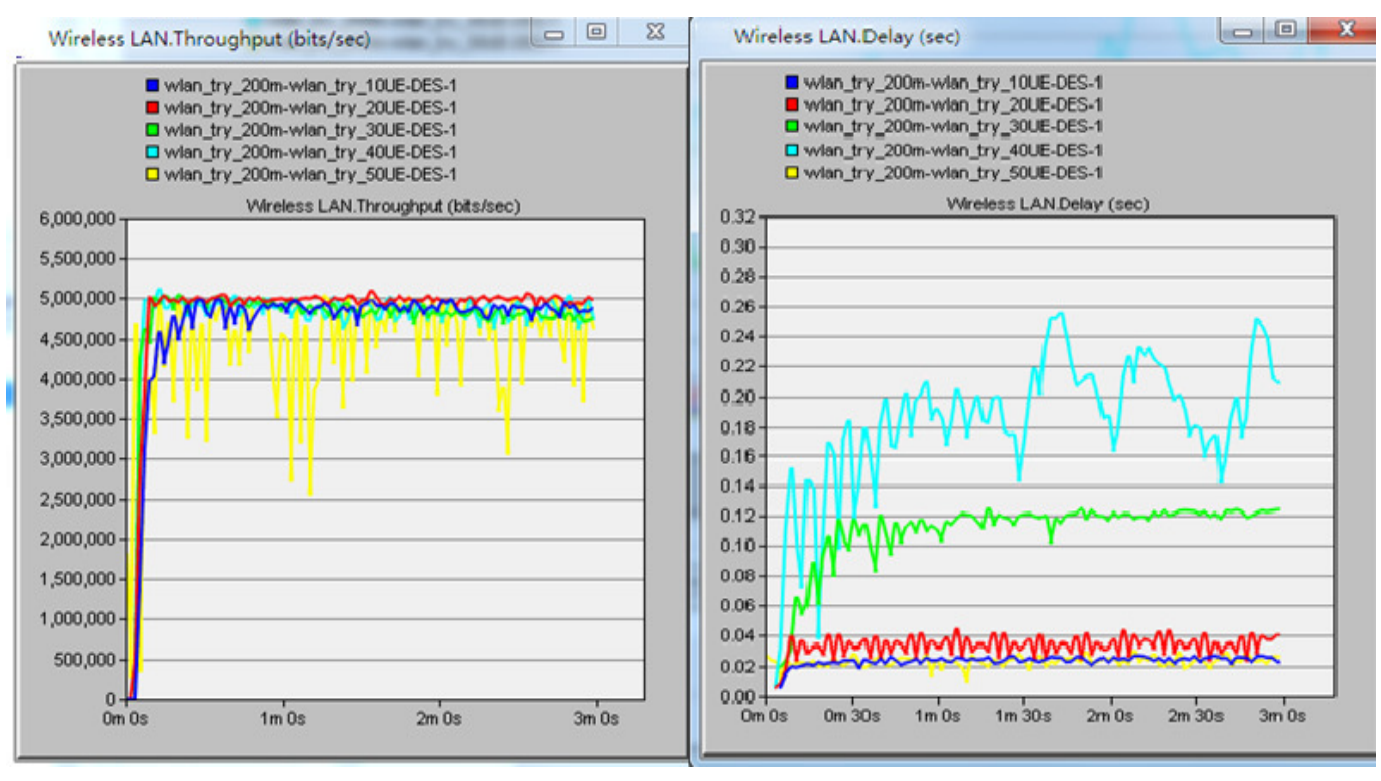

Figure 7. The throughput and delay contrast of a BSS with different Users
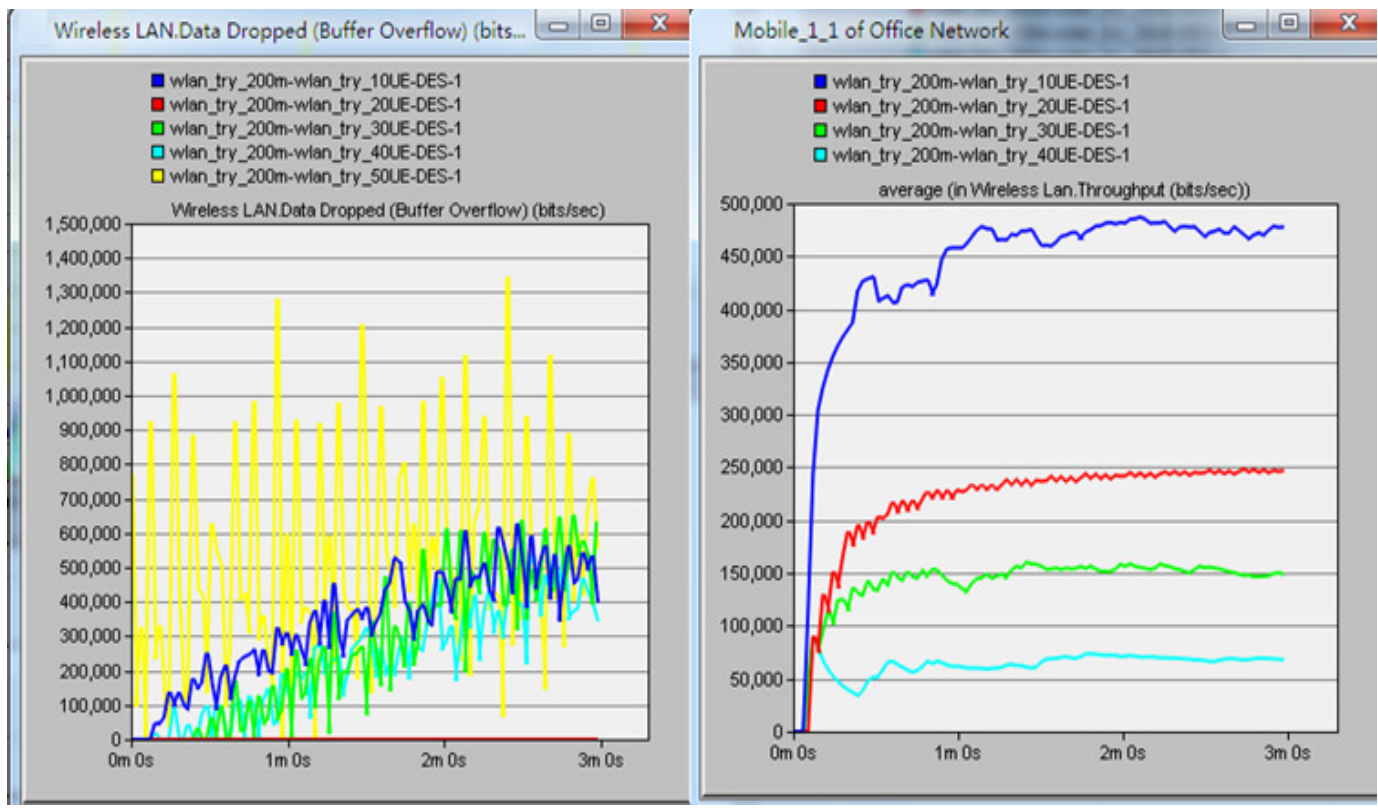

Figure 8. The data dropped size and throughput of one UE contrast with different Users

Figure 8 depicts the total throughput, the time delay, the data dropped, and the throughput of a single user's under every circumstance. It is observed that the total throughput of the network is about 5.1Mbps, the time delay and the data dropped are increasing, and the throughput of a single user's is decreasing when the network becomes a dense one. That is to say, optimal configuration can improve the WLAN networks carrying capacity and make it more efficiently. 


\subsection{A test of the BSS's Throughput}

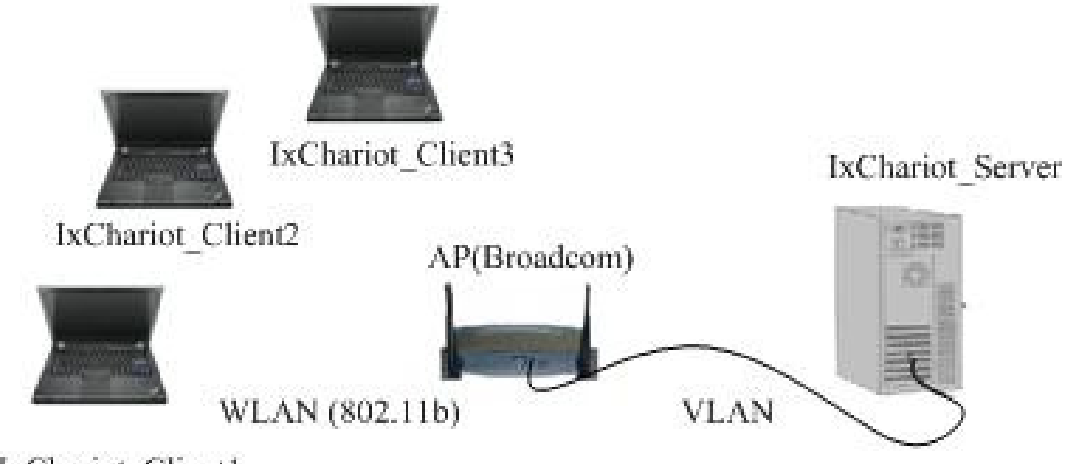

IxChariot_Client1

Figure 9. A Scene of a BSS's throughput test

For testing a BSS's throughput, a small scale $802.11 \mathrm{~b}$ network was set up in the office environment like figure 9. Ixchariot_Client is the ftp client fixed on PC, Ixchariot_Server is the ftp server fixed on server, AP is connected with sever by VLAN, and PC is connected with AP by 802.11b WLAN network. In this case, network coverage is out of our consideration, so free-space attenuation is supposed for the test thus the loss is a little larger indeed. At first, we let one Ixchariot_Client link the AP by downloading the package of $1 \mathrm{MB}$ bytes in MAC layer. Other network configuration looks like the table 4.

Table 4. Parameters configuration in the test of the BSS's Throughput

\begin{tabular}{|l|l|l|l|l|}
\hline Parameters & $\begin{array}{l}\text { BSS } \\
\text { Number }\end{array}$ & $\begin{array}{l}\text { AP } \\
\text { Number }\end{array}$ & $\begin{array}{l}\text { UE } \\
\text { Number }\end{array}$ & service \\
\hline Values & 1 & 1 & $1 / 6$ & FTP \\
\hline Parameters & $\begin{array}{l}\text { DATA } \\
\text { Length }\end{array}$ & Time & Power & $\begin{array}{l}\text { Access } \\
\text { Mechanism }\end{array}$ \\
\hline Values & 640KB & $0.55 \mathrm{~s}$ & $100 \mathrm{~mW}$ & CTS_self \\
\hline
\end{tabular}

The largest throughput of the BSS can be gotten in figure 10. That is to say the maximal throughput of MAC layer is nearly 7.0Mbps, a little less than simulation result. Then we let six Ixchariot_Clients communicate with the AP simultaneously, we can obtain the total largest throughput of MAC layer is nearly $6.6 \mathrm{Mbps}$ in figure 11 , a little less than the single user scene because of channel accessing competition between multiple users. 
International Journal of Computer Networks \& Communications (IJCNC) Vol.5, No.6, November 2013



Figure 10. The throughput a BSS's throughput test with $1 \mathrm{UE}$

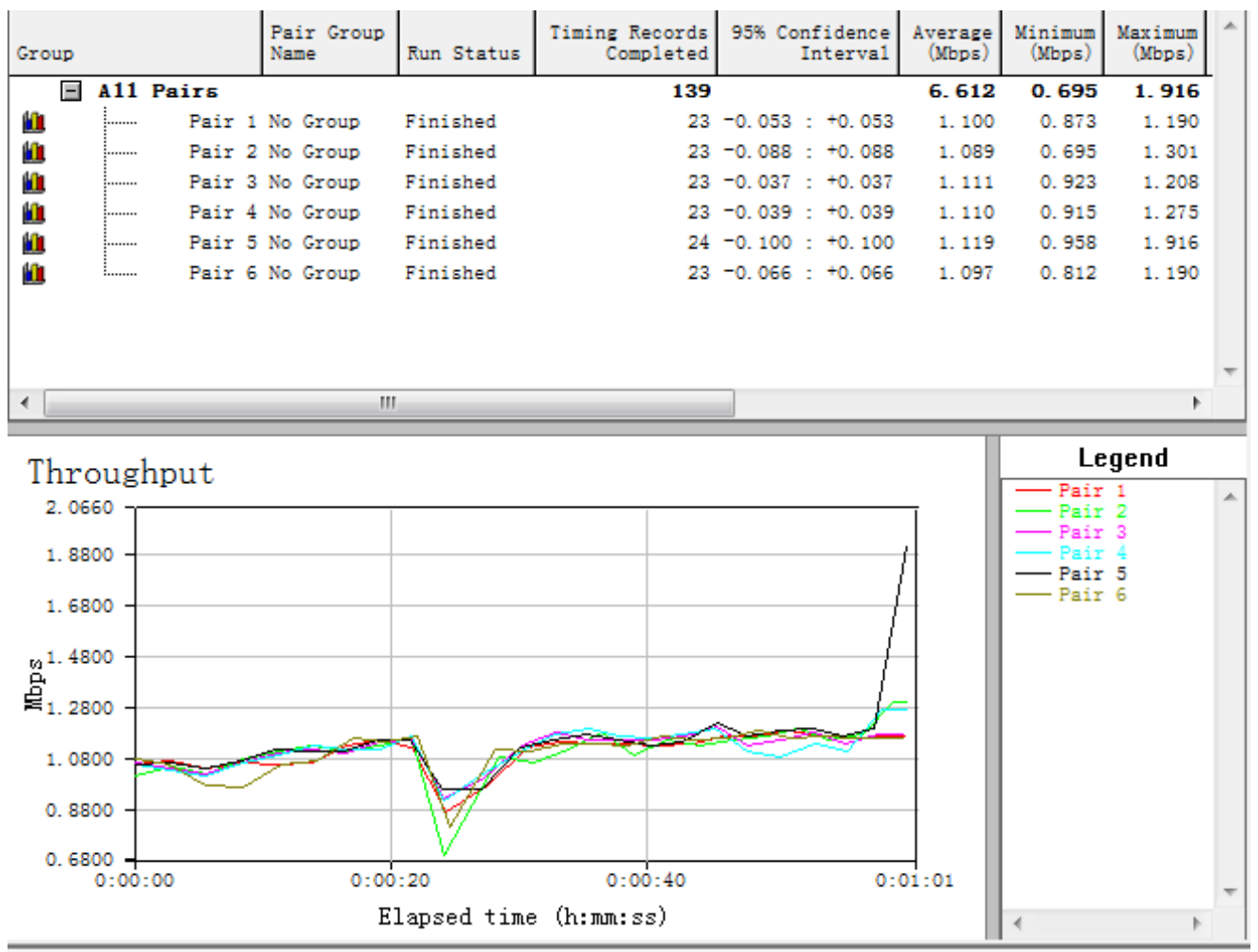

Figure 11. The throughput a BSS's throughput test with 6 UE 
International Journal of Computer Networks \& Communications (IJCNC) Vol.5, No.6, November 2013

\subsection{SIMULATION D: Two BSS's Throughput Contrast with TCP Protocol}

As we know, $802.11 \mathrm{~b}$ uses DSSS modulation to support the data rate $11 \mathrm{Mbps}$. DSSS system can make the narrow-band noise broaden so as to decrease interference brought by other wireless systems in the same bands. To evaluate the interference, we suppose a scenario with two WLAN networks like figure 12 .

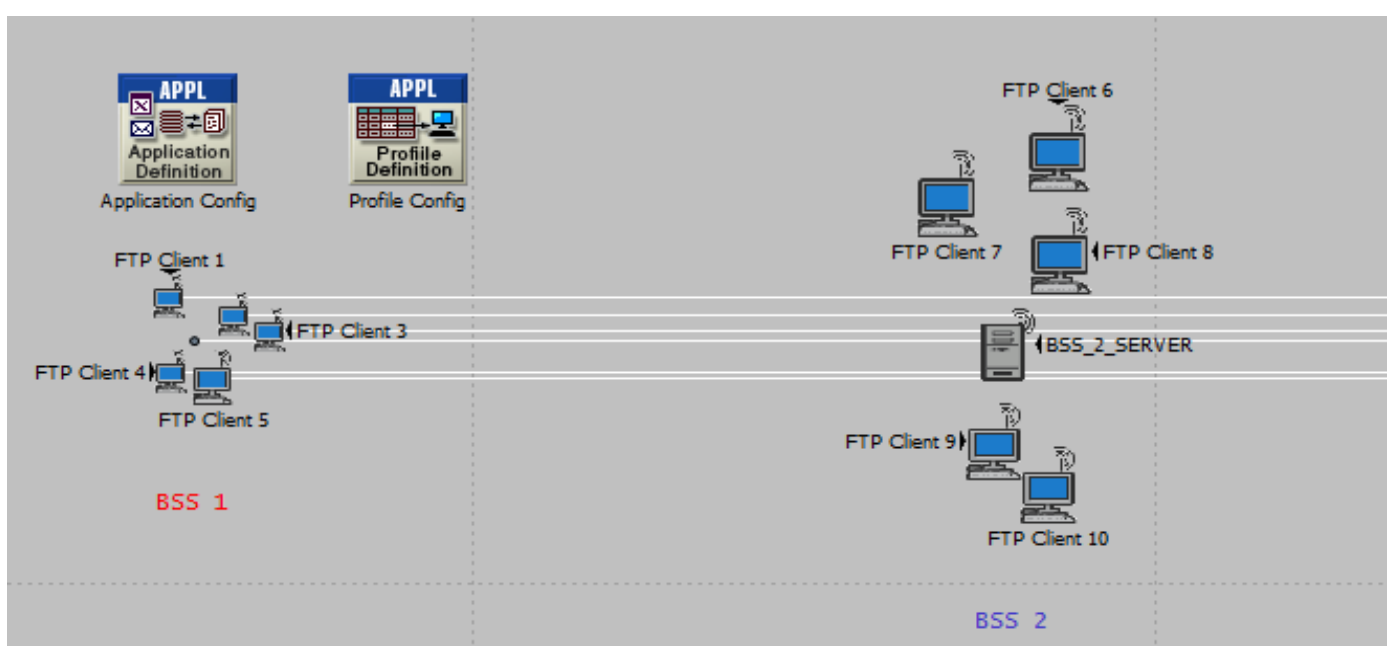

Figure 12. The scene of two BSS's throughput simulation

Presumption is made as every 5 UEs accessing the same channel in one BSS and assuming every 5 UEs randomly located in one cell. The users' transmitting speed is 11Mbps and CTS_self protocol are used in MAC layer. The free space model is used to calculate path loss, the users' transmitting power is $100 \mathrm{~mW}$ and all UEs and Aps both in BSS1 and BSS2 are set to use channel $1 /$ channel 1 to communication separately.

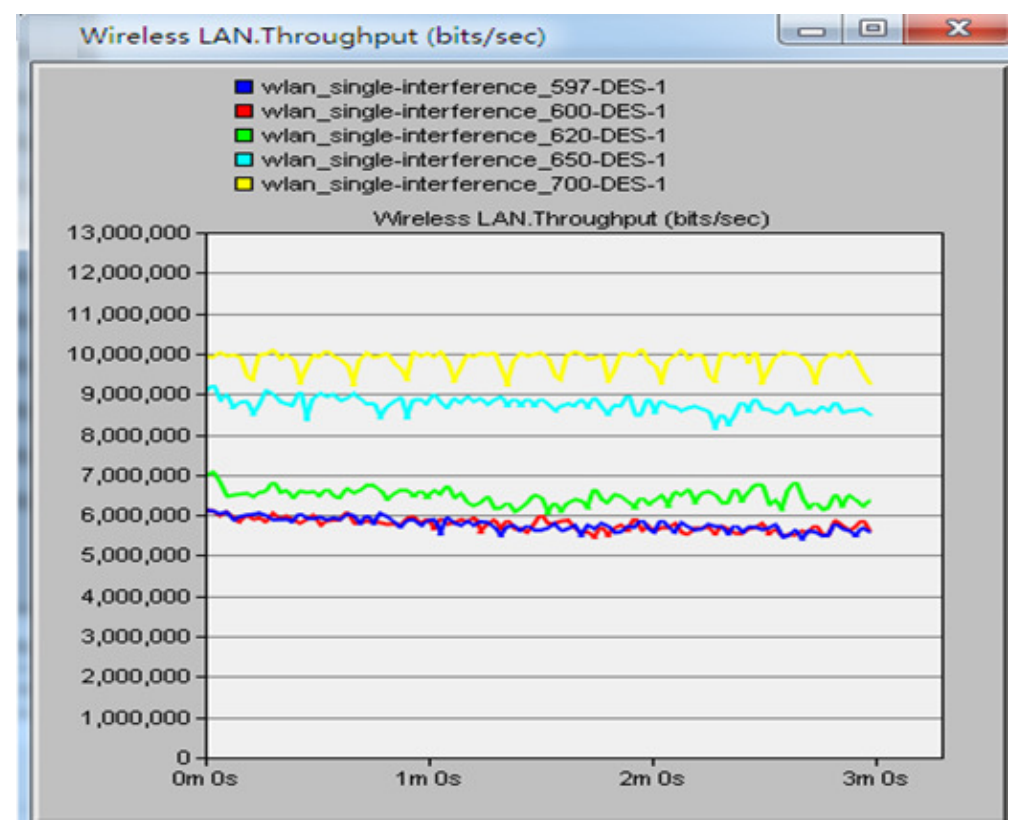

Figure 13. The total throughput contrast of two BSS's with different distance 


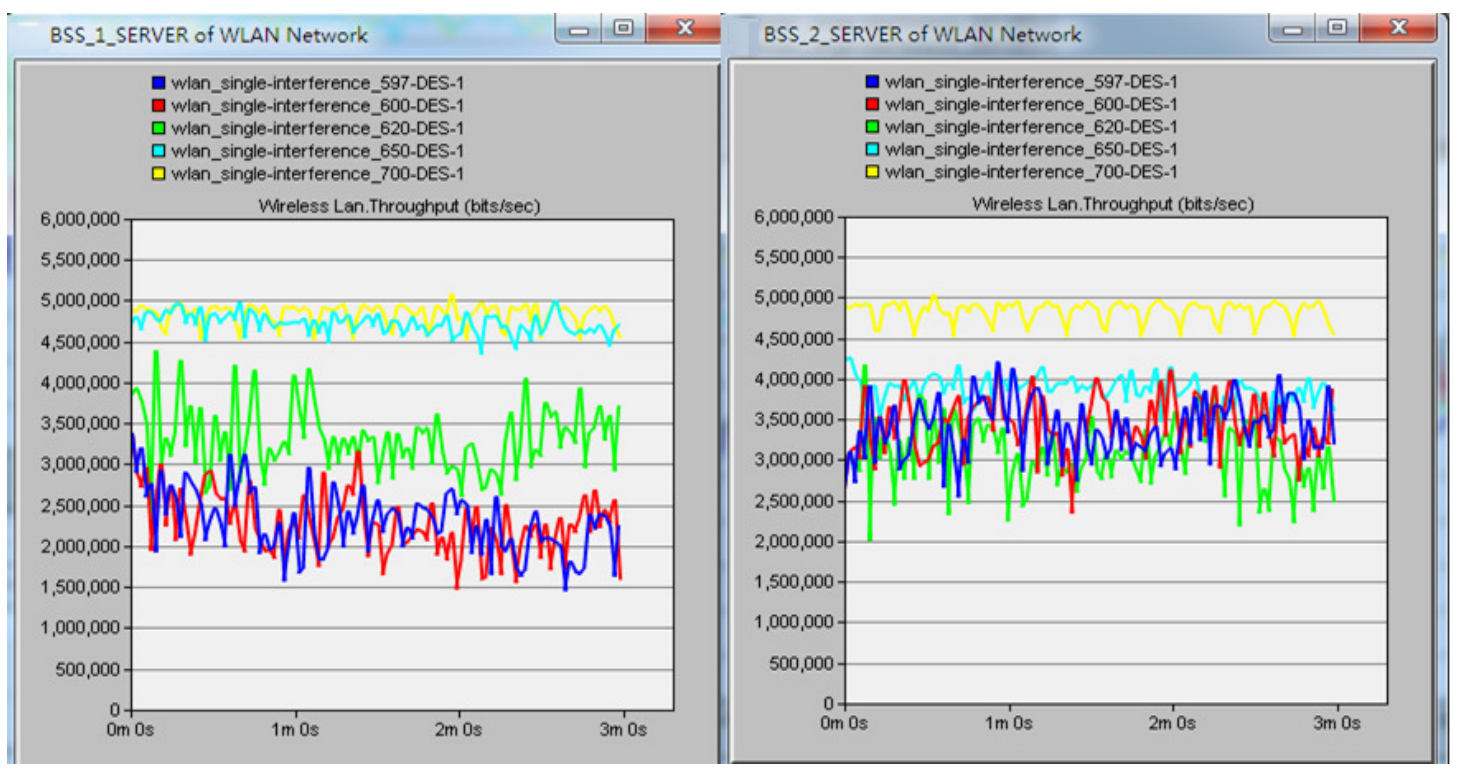

Figure 14. Each BSS's throughput contrast with different distance

Here, we want to check one AP's coverage to set two APs' being parted $597 \mathrm{~m}, 600 \mathrm{~m}, 620 \mathrm{~m}$, $650 \mathrm{~m}$ and $700 \mathrm{~m}$. In figure 13, the total throughput is about 500, 500, 580, 600 and $1200 \mathrm{~K}$ bytes of two BSS's, which shows the interference is serious when the channel is overlapped and the interference becomes seriously when the distance is closer. And each BSS's throughput with different distance is plotted in the figure 14, which confirms that additional RF medium contention overhead occurs for all radios using the same channel in physical area resulting in throughput degradation and latency.

\section{Conclusions}

In this paper we have proposed much analysis, many simulation cases and real tests to illustrate the performance of $802.11 \mathrm{~b}$ MAC layer, application layer and the fairness issues related to DCF. Let us recall here that our final goal is to suggest the details which should be paid attention to in deploying network services. First, with the constraint of DCF, the largest user number is less the 40 in a BSS (Actually, 20 users are much better in a BSS with $802.11 \mathrm{~b}$ or $802.11 \mathrm{~g}$ WLAN network). Second, the condition of DCF is that all users have the same channel condition. That is to say those WLAN network operators have to find other method to achieve loading balance for users with different channel condition in real circumstance. And the last contribution of this paper is the simulation results and test results are general values which can be offered as the basis.

\section{ACKNOWLEDGEMENTS}

This work is supported by Important National Science and Technology Specific Projects NO.2013ZX03003016. 
International Journal of Computer Networks \& Communications (IJCNC) Vol.5, No.6, November 2013

\section{REFERENCES}

[1] Lee, S.hyun. \& Kim Mi Na, (2008) "This is my paper", ABC Transactions on ECE, Vol. 10, No. 5, pp120-122. IEEE Computer Society, (1999) "Wireless LAN Medium Access Control (MAC) and Physical Layer (PHY) Specifications”, IEEE Std. 802.11-1999.

[2] Soung Chang Liew, Ying Jun Zhang, (2008) "Proportional Fairness in Multi-channel Multi-rate Wireless Networks C Part I: The Case of Deterministic Channels with Application to AP Association Problem in Large-Scale WLAN",IEEE Trans. on Wireless Communications, Vol.7, No.9, pp.34463456.

[3] G. Bianchi, (200) "Performance analysis of IEEE 802.11 distributed coordination function, IEEE J. Select. Areas. Commun.”, Vol. 18, No. 3, pp.535-547.

[4] Xiang Ling and Kwan Lawrence Yeung, (2006) "Joint Access Point Placement and Channel Assignment for 802.11 Wireless LANs" IEEE Trans. on Wireless Communications, Vol.5, No.10, pp. 2705-271.

[5] A. Hills, (2001) “Large-scale wireless LAN design”, IEEE Commun.Mag., Vol.39, No.11, pp.98-107.

[6] G. Anastasi, E. Borgia and M. Conti, E. Gregori, (2005) "IEEE 802.11b Ad Hoc Networks: Performance Measurements", Cluster Computing, Vol 8, pp.2-3.

[7] Purvang Dalal, Nikhil Kothari and K. S. Dasgupta, (2011)“Improving TCP Performance over Wireless Network with Frequent Disconnections," International Journal of Computer Networks \& Communications (IJCNC) Vol.3, No.6, pp.169-184.

\section{Authors}

Mingming Li works in SRRC of China as an engineer, ever graduated from Beijing University of Post and Telecommunication in 2011. Her research field is frequency spectrum planning and spectrum demand forecasting 